\title{
Berichte von der Kurie über den Erwerb umstrittener Prozeßmandate und Privilegien (12.-13. Jahrhundert)
}

\section{Dietrich LohrmanN}

Die Neuigkeiten von der Kurie (Nova curiae), die ich auf dem Programm der Tagung angekündigt habe, hätte man im älteren Deutschen des 17.-18. Jahrhunderts als ,Zeitungen vom Papsthof' bezeichnet. Solche Mitteilungen betrafen unmittelbar aktuelle Vorkommnisse aus dem Umfeld der Kurie, die in Europa an interessierter Stelle verbreitet wurden. Sie sind aus der neueren Geschichte, wie es scheint, in größerer Menge erhalten. Fritz Kern in seinen «Acta Imperii Anglie et Francie» nennt bereits aus dem späteren 13. und dem 14. Jahrhundert eine ganze Reihe von Beispielen ${ }^{1}$. Für die Frühzeit sind sie selten und weit verstreut. Über die entscheidenden Verhandlungen am Papsthof und ihren Verlauf, wie insbesondere über die Reaktionen der Päpste auf den jeweiligen Vortrag der Kläger und Bittsteller, erfahren wir dadurch in der Regel nur Weniges. Für das Regestenwerk Italia pontificia ist das nicht gravierend, denn dort sind Verhandlungen vor dem Papst nicht als Regesten vorgesehen. Sie gehören weder in den Band Rom noch in die entsprechenden Bände der sonstigen Aufenthaltsorte der Kurie. Trotzdem liefern solche Berichte für das Verständnis vieler Entscheidungen sehr wesentliche Gesichtspunkte. Sie lesen sich auch viel lebhafter als die Urkunden selbst und verdienen daher gesammelt zu werden.

Petitionen oder Entwürfe von Urkunden, aus deren Varianten gegenüber den Ausfertigungen wir Erkenntnisse über die Vorverhandlungen ableiten könnten, sind bekanntlich ebenfalls nur selten erhalten ${ }^{2}$. Die am Ende ausgefertigten Papsturkunden liefern in der Narratio zwar knappe zusammenfassende Angaben (etwa so: die streitenden Parteien seien aufmerksam angehört worden, und der Papst entscheide nun nach Beratung durch seine fratres, die Kardinäle), aber Näheres ist auch daraus nicht zu entnehmen. Erst ab 1198 geht es dank den Registerbänden Innozenz'III. des öfteren auch in die verfahrensrechtlichen

1 Fritz Kern: Acta Imperii Anglae et Franciae ab anno 1267 ad annum 1313, Tübingen 1911 (ND 1973), S. VIII: „Es gehört zu den seltenen Freuden heutiger Archivarbeit, solchen frischen Zeugnissen des 13. Jahrhunderts ... zu begegnen“. Die von Kern edierten Berichte (Nr. 2, 8, 19, 38, 48) gehören in die Jahre 1273 bis 1284.

2 Beispiele bei Dietrich Lohrmann: Kirchengut im nördlichen Frankreich. Besitz, Verfassung und Wirtschaft im Spiegel der Papstprivilegien des 11.-12. Jahrhunderts, Bonn 1983 (Pariser Historische Studien 20), S. 90-107. 
Details ${ }^{3}$. Ich überblicke freilich bislang nur einen Teil des französischen Materials, vor allem das nordfranzösische, und kann nicht sagen, was sich bei näherer Prüfung aus den Angaben der Italia pontificia oder den Papstregistern des 13. Jahrhunderts für Italien ergeben würde.

Nicht selten ist schon im 11. Jahrhundert von einer audientia domini papae die Rede, einem Begriff, der vielfach bereits untersucht und schon aus der älteren Zeit wohlbekannt ist ${ }^{4}$. Zitiert sei nur ein früher Brief Gregors VII. aus dem Jahre 1073 an den Erzbischof Manasses von Reims, dieser möchte es nicht länger zulassen, daß die Papstaudienz durch zu viele Klagen bemüht werde: totiens apostolicam audientiam fratrum clamoribus fatigari. Die Kurie widersprach damit nicht ihren sonstigen Erklärungen, in denen sie sich weitherzig zur Zuflucht aller anbot, die Recht und Schutz suchten. Der Erzbischof von Reims soll nur sein persönliches Verhalten ändern und die Zahl begründeter Klagen nicht unnötig anschwellen lassen.

Vier Jahre später reiste Erzbischof Manasses von Reims selbst nach Rom und suchte seine Exkommunikation und Suspension durch den übereifrigen Legaten Hugo von Die rückgängig zu machen. Nach elfwöchigem Warten kam es endlich zur Verhandlung vor dem Papst und einer römischen Fastensynode. Manasses' offensive Strategie gegen den Legaten trug hier offensichtlich Früchte,

3 Die Register Papst Innocenz'III., 8: 8. Pontifikatsjahr 1205/1206, ed. Othmar Hageneder/Andrea Sommerlechner Wien 2001 (Publikationen des Historischen Instituts beim Österreichischen Kulturinstitut in Rom 2/8), S. 351-357 Nr. 205 vom 18. Jan. 1206: Cum igitur ...super monasterii vestri subiectione ac libertate controversia verteretur et nos eam examinandam commisissemus iudicibus delegatis, ipsi causam eandem sufficienter instructam ad nostram presentiam remiserunt, certum partibus terminum prefigentes, quo cum instrumentis et attestationibus nostro se conspectui presentarent sententiam recepture. Partibus igitur in nostra presentia per procuratores idoneos constitutis audientiam prebuimus liberam et benignam. Siehe dazu den unten Anm. 33 angezeigten Bericht des Sachwalters einer der Parteien (Prozeß Evesham gegen Bischof von Worcester).

4 Peter Herde: Beiträge zum päpstlichen Kanzlei- und Urkundenwesen im 13. Jahrhundert, Kallmünz ${ }^{2} 1967$, S. 213-223. In dieser verbesserten und erweiterten Auflage sind die Ergebnisse von Herdes Edition der Formelbücher eingearbeitet, Peter Herde: Audientia litterarum contradictarum. Untersuchungen über die päpstlichen Justizbriefe und die päpstliche Delegationsgerichtsbarkeit vom 13. bis zum Beginn des 16. Jahrhunderts, Tübingen 1970 (Bibliothek des Deutschen Historischen Instituts in Rom 31-32), S. 223-238. Weitere Literatur in den Artikeln ,Audientia litterarum contradictarum " und ,Audientia sacri palatii' von Peter Herde und Gero Dolezalek im: LexMA 1 (1980) Sp. 1192-94. Zur früheren Zeit (Gregor I.) vgl. Ernst Pitz: Papstreskripte im frühen Mittelalter. Diplomatische und rechtsgeschichtliche Studien zum Brief-Corpus Gregors des Großen, Sigmaringen 1990 (Beiträge zur Geschichte und Quellenkunde des Mittelalters 14), S. 243-246, und Hans Gabelmann: Antike Audienz- und Tribunalszenen, Darmstadt 1984.

5 Das Register Gregors VII., hg.v. Erich CAspar, Berlin 1920/1923 (MGH Epp. sel. 2/ 1-2), I/13 S. 21. Mit den fratres sind auswärtige Bischöfe als Kläger gemeint. Das Register enthält zahlreiche andere Belege für apostolica audientia (Index S. 677a unten). 
trotz deutlicher Bedenken Gregors VII., denn der ihm lästige Manasses, den er als selbstbewußten Vertreter des älteren Systems nur schwer ertragen konnte, wurde wieder eingesetzt. Die anwesenden Kardinäle, auf deren Wunsch der Beschluß offenbar erfolgt war, hatten sich beeindrucken lassen durch die Klagen des Manasses und die Beschwerden mehrerer anderer französischer Erzbischöfe, die ebenfalls durch den Legaten suspendiert worden waren. Widerwillig entschloss sich deshalb auch Papst Gregor zur Suspension der Suspensionen, unter der Bedingung freilich, daß sämtliche Beklagten sich noch vor dem Legaten zu entschuldigen hätten. Eine Desavouierung des Gregor eng vertrauten Legaten Hugo von Die war so vermieden ${ }^{6}$.

Beschränken wir uns nun auf die subjektiven Berichte aus der Sicht einzelner Prozeßparteien. Sie bilden die einzige Quellengruppe, die gelegentlich den Verlauf einer mündlichen Verhandlung vor Papst und Kardinälen genauer schildert. Nur in einem Sonderfall liegt für die Jahre 1148-1152 der Bericht eines mehr oder weniger neutralen Dritten vor, Johannes von Salisbury. Er hat als Augenzeuge einige Jahre später eine ganze Folge aufschlußreicher, zum Teil geradezu anrührender Szenen von Verhandlungen vor Konzilien bzw im Konsistorium festgehalten ${ }^{7}$.

\section{Französische Berichte aus dem 12. Jahrhundert}

Im 12. Jahrhundert gibt es, wie angedeutet, noch keine institutionell verfestigte Instanz, die es den streitenden Parteien erlaubt hätte, Einspruch gegen die Ausfertigung einer Papsturkunde zu erheben, welche einen der Prozeßgegner begünstigte. Von contradictio ist zwar die Rede, doch die dringend notwendige

6 Register Gregors VII. (wie Anm. 5) V/17 S. 378-380; dort auch der Eid, den Manasses zu schwören hatte. Der Papst betont ausdrücklich: non sine gravi labore discussimus. Die näheren Umstände des Aufenthaltes an der Kurie und der Verhandlung erfahren wir aus Manasses' zwei Jahre späterem Brief an den Legaten bei Bouquet 14 S. 782: tandem in praesentia domni Apostolici et in concilio generali inter nos et eos qui ibi loco vestro ... aderant, altercatio habita est, et ex eorum accusatione ac nostra defensione quidquid passi eramus temere ac violenter actum esse et constare non debere, judicatum atque correctum est. Tum ego domno Apostolico renunciavi cunctis audientibus. Ipso autem Apostolico subsequenter interrogante. Deinde etiam idem instituit domnus Apostolicus. Die Überlieferung des nachfolgenden Verfahrens gegen Manasses siehe bei Dietrich Lohrmann: Papes et cardinaux en France et en Bourgogne au temps de la Réforme grégorienne, in: L'abbaye d'Ainay des origines au XIIe siècle, dir. Jean-François Reynaud/François Richard, Lyon 2008, S. 161 - 179. In den Briefsammlungen der Zeit Heinrichs IV., ed. Carl ErdmanN/ Norbert Fickermann, Weimar 1950 (MGH Epp. DK 5), S. 243 f., findet sich die Beschreibung einer weiteren Absetzungsverhandlung 1075 an der Kurie, hier aus Bamberger Sicht: Ecce autem Rome, dum terminum vocationis sue supersedisset ...

7 Siehe weiter unten. 
Instanz einer audientia litterarum contradictarum hat erst Innozenz III. kurz nach 1200 geschaffen $^{8}$. Wichtige Rechtsfragen und Einsprüche waren somit in der früheren Zeit vom Papst selbst zu prüfen. Das führte zu Überlastung und gelegentlich auch Fehlentscheidungen oder Urkunden, die ohne Wissen des Papstes herausgingen. Zuweilen, so insbesondere bei Erschleichung von Urkunden mittels Vorspiegelung falscher Tatsachen, beklagten die Päpste selbst den Mißstand: die Fülle der Geschäfte habe sie überfordert, es sei daher zu einem Fehler gekommen?.

Erstes Beispiel: 1107, als Papst Paschalis II. - kein erfahrener Jurist - nach Frankreich kam und bereits in Dijon angelangt war, erhob dort ein angesehener Gelehrter, der Magister Anselm von Laon, Einspruch gegen die Wahl eines neuen Bischofs seiner Stadt. Der Elekt war Kanzler des Königs von England gewesen. Er hatte sich in dieser Funktion massiv bereichern können und war persönlich, glaubt man dem Berichterstatter, vor allem an militärischen Dingen und Fragen der Jagd interessiert. Von kirchlichen Weihegraden preter clericatum war er noch unberührt, die Diakonsweihe nahm er erst nach seiner Wahl entgegen. Mehr lag ihm an der Verbreitung der Kunde von seiner Zahlungsfähigkeit, und damit stieß er am Papsthof auf offene Ohren. Auch die bedürftigen capellani papae und zumindest ein Teil der Kardinäle zeigten sich empfänglich ${ }^{10}$.

Unser Bericht stammt von keinem Unbekannten. Er steht in der Autobiographie eines Abtes der Diözese Laon, Guibert von Nogent ${ }^{11}$. Genau erzählt dieser, wie der Papst ihn zusammen mit zwei anderen Äbten zur Person des Elekten persönlich befragt hatte. Seine Antworten bezeichnet er selbstkritisch als lauwarm, wenn auch nicht ganz falsch: verba tepentia, sed non penitus a vero deviantia. Mit Bestimmtheit bejahen konnte er nur die Frage, ob der Elekt

8 Herde: Audientia (wie Anm. 4). Über die Begriffe consistorium and consilium fratrum nostrorum (Beratung mit den Kardinälen) vgl. die instruktive Untersuchung von Sarah Noethlichs: Das päpstliche Konsistorium im Spiegel der Quellen des 11.-12. Jh., in: ZRGKanAbt 94 (2008) S. 272-287.

9 Vgl. u.a. Herde: Beiträge (wie Anm. 4) S. 93 f., 110 Anm. 197: per falsi suggestionem et veri suppressionem; weiteres ebenda S. 63, 112 f. Vgl. auch Lohrmann: Kirchengut (wie Anm. 2) S. 67 f. ; zu Gregor VII. Pitz: Papstreskripte (wie Anm. 4) S. 56 und Index s. v. Erschleichen. Das reiche Material zu Erschleichungen im 12. Jahrhundert kann ich hier nicht ausbreiten. Sehr aufschlussreich schon der Bericht des Bischofs Hugo von Grenoble zum Streit mit Erzbischof Guido von Vienne um den Pagus von Sermorens und seine Burgen vor Papst Urban II., analysiert von Beate Schilling in GalP 3/1 S. 136-144 Nr. *183-*209. Weiteres bei Alfons BeCker: Päpstliche Gerichtsurkunden und Prozessverfahren zur Zeit Urbans II. (1088-1099), in: Zwischen Saar und Mosel. Festschrift für Hans-Walter Herrmann, hg.v. Wolfgang Haubrichs al., Saarbrücken 1995, S. 39-48.

10 Guibert de Nogent, Histoire de sa vie (1053-1124), ed. Georges Bourgin, Paris 1907, S. 141-143; ders., Autobiographie, ed. Edmond-R. Labande, Paris 1981, S. 286-292. Dazu François Dolbeau: Deux nouveaux manuscrits des «Mémoires» de Guibert de Nogent, in: Sacris Erudiri 26 (1983) S. 155-176.

11 Guibert, ebd. 
rechtmäßiger Geburt sei. Aber auch hier zierte er sich: der Elekt sei neque nothus neque spurius. Klagen oder Einwände in offener, eindeutiger Form äußerte er nicht. Angesichts der vorherrschenden Stimmung unter den Kurialen zugunsten des zahlungskräftigen Kandidaten resignierte jedoch nicht nur er, der kleine Abt. Selbst der mutige Magister Anselm, der zuvor offen Klage geführt hatte, verzichtete im Laufe der weiteren Verhandlung darauf, „dem Herkules (gemeint ist der Elekt) die Keule aus der Hand zu reißen“. Guibert seinerseits wird am Schluß von einer Gruppe Kardinäle wegen seiner gewundenen Erklärungen beglückwünscht: Multum placuerunt nobis verba tua. Er schämt sich, denn er hat es nicht verhindert, daß nun das Bischofsamt, die gratia pontificandi, an einen völlig ungeeigneten Kandidaten gelangt.

Dem neuen Bischof Galterus (Gautier) von Laon sind selbstverständlich die Urkunden und Briefe ausgefertigt worden, die zum Amtsantritt eines Bischofs üblich waren. Das wird in dem literarisch überformten Bericht des Guibert von Nogent nicht ausdrücklich hervorgehoben. Sein Fazit lautet viel allgemeiner: „Geld beherrscht alles, so auch an der Kurie. Wenn keiner ein offenes Wort wagt, wie leider auch ich es getan habe, kommen die falschen Leute in die höchsten Ämter, und das zieht furchtbare Folgen nach sich.“

Der zweite Bericht ist viel kürzer gehalten. Er stammt aus dem Benediktinerkloster Saint-Bertin in der nordfranzösischen Diözese Thérouanne. Diese bedeutende Abtei geriet während der ersten Hälfte des 12. Jahrhunderts in eine schwierige Lage, da sie im Zuge der flandrischen Klosterreform der Oberhoheit des Abtes von Cluny unterstellt worden war. Energisch versuchte man, die alte Freiheit zurückzugewinnen. Die «Gesta abbatum Sancti Bertini» liefern in diesem Zusammenhang eine Reihe von Nachrichten über Romreisen und Appellationen vor verschiedenen Päpsten. Es geht um den Erwerb von Privilegien für die eine wie für die andere Seite und schließlich auch, 1130, um allgemeinere Bemerkungen über das päpstliche Schisma. Besonders aufschlußreich wirkt eine Notiz, die wie unser erstes Beispiel aus Laon noch in die Zeit des leicht zu überzeugenden Mönchspapstes Paschalis II. gehört: Animatus itaque suorum consilio reist der Abt Lambert von Saint-Bertin 1112 nach Rom. Er will an der Kurie die unberechtigten Ansprüche der Cluniazenser darlegen: expositurus Romane curie Cluniacensium iniuriam. Das Glück (fortuna) lächelt ihm so freundlich $\mathrm{zu}, \mathrm{da}$ ihm alle Anträge problemlos (absque ulla difficultate) von Papst und Kurie bewilligt werden. Die Stimmung dort, bemerkt der Text, war gerade stark anticluniazensisch geprägt. Leider nennt er dafür nicht die Gründe, man mag sie in den beginnenden Schwierigkeiten des Abtes Pontius suchen ${ }^{12}$.

12 Gesta abbatum S. Bertini Sithiensium, ed. Oswald Holder-Egger, in: MGH SS 13, Hannover 1881, S. 643 f. Zu Abt Pontius vgl. Gerd Tellenbach: Der Sturz des Abtes Pontius von Cluny und seine geschichtliche Bedeutung, in: QFIAB 42/43 (1963) S. 1355, sowie Neithard Bulst: Art. Pontius 1, in: LexMA 7 (1995) Sp. 98. 
Auch die erworbenen Urkunden sind nicht näher aufgeführt ${ }^{13}$. Aufschlußreicher wirkt, daß der Abt von Saint-Bertin seinem Erfolg selbst nicht traut. Er fürchtet einerseits Romanorum cupiditatem et inconstantiam, zum anderen die cluniazensische constantiam. Cluny läßt sich nämlich auf keinerlei Friedensangebot ein und sendet Boten auf Boten nach Rom, um die Gunst des Papstes zurückzugewinnen ${ }^{14}$.

Wieder unmittelbar in die Einzelverhandlung vor dem Papst, nunmehr 1141 vor Innozenz II., führt unser drittes Fallbeispiel. Es ist ein Bericht über die Romreise des Abtes Hariulf von St. Peter in Oudenbourg (Diözese Tournai). Dieser Text ist nicht unbekannt, aber trotzdem so schön, daß ich ihn hier unmöglich übergehen kann. Dem bereits greisen Abt im schlohweißen Haar hatte sein Gegner, der Abt von Saint-Médard in Soissons, wirksamen Widerstand gegen ein perfides Übernahmemanöver nicht mehr zugetraut; der Alte lasse bereits die Flügel hängen. Frech hatte der Abt aus Soissons sich deshalb ein Schreiben des Papstes verschafft, das den Abt Hariulf kurzerhand aufforderte, sich der Autorität des Abtes von Saint-Médard zu unterstellen. Statt feindlicher Übernahme an der Börse wie in unserer Zeit investierte man in der ersten Hälfte des 12. Jahrhunderts, um sein Ziel zu erreichen, an der Kurie und fügte notorisch falsche Angaben hinzu wie die, Abt Hariulf sei selbst zuvor ein Mönch von Saint-Médard in Soissons gewesen ${ }^{15}$.

Der wackere Greis ermannte sich jedoch. Er nahm alle Mühen der Reise auf sich und erschien, empfohlen durch Briefe des Bischofs von Noyon-Tournai sowie des Abtes Bernhard von Clairvaux, unvermittelt vor dem päpstlichen Kanzler Haimerich. Der verschaffte ihm Zugang zum Papst. Vorauf gingen weitere Gespräche mit mehreren Kardinälen. Um sich vor dem Papst in angenehmer Weise zu präsentieren und nicht gleich mit der Tür ins Haus zu fallen, beginnt unser flandrischer Abt mit einer Fabel: Ein Mann heiratet die ,Exfreundin' eines Rivalen und lebt 35 Jahre lang mit ihr zusammen. Da erscheint der Rivale und will die Frau entführen, da sie ihm als seine Verlobte gehöre. In dieser Weise, so Abt Hariulf zu Innozenz II., sei auch der Abt von Saint-Médard vorgegangen, und der Papst habe ihn dabei unterstützt, indem er den entsprechenden Brief ausstellen ließ. Indes, Nemo absens condempnetur, zitiert Hariulf den sogen. Zephyrinus (Pseudo-Isidor), einen Text, der kurz darauf

13 Gesta, ed. Holder-Egger (wie Anm. 12) S. 643.

14 Mehr zu ermitteln, bleibt eine Aufgabe der Gallia Pontificia. Vgl. einstweilen Laurent Morelle: Par delà le vrai et le faux: trois études critiques sur les premiers privilèges pontificaux de l'abbaye de Saint-Bertin (1057-1107), in: L'acte pontifical et sa critique Études réunies par Rolf Grosse, Bonn 2007 (Studien und Dokumente zur Gallia Pontificia 5), S. 51-86.

15 Ernst Müller: Der Bericht des Abtes Hariulf von Oudenburg über seine Prozessverhandlungen an der römischen Kurie im Jahre 1141, in: NA 48 (1930) S. 97-115. 
auch in der Rechtssammlung Gratians erscheint (C. 3 q. 9 c. 13) ${ }^{16}$. Solche Kritik behagte dem Kanzler Haimerich gar nicht, er schaltet sich ein: Domne abbas, videte quomodo caute loquamini! Doch das bringt den Greis erst wirklich in Fahrt: iste littere ... (gemeint ist der Papstbrief) omni dolo, omni mendacio omnique fallacia plene fuerunt. Im höchsten Maße sei er darüber empört und verwirrt gewesen, daß diese littere ... omni veritate carebant et tamen domini apostolici bullam habebant.

Wichtig ist nachfolgend die Vorgehensweise bei der Auswahl der delegierten Richter, die den Sachverhalt untersuchen sollen. Wir erhalten hier sogar wörtliche Rede. Für die Untersuchung schlägt man dem ungestümen Abt Hariulf drei mögliche Richter vor. Darunter sind zwei Bischöfe, die er in folgender Weise ablehnt: Der Bischof von Châlons sei ein ehemaliger Mönch von SaintMédard, also befangen, und der Bischof von Arras habe die ganze Belästigung persönlich angezettelt. Konsequenz für den Abt: Habet Francia alios viros, non inferioris gracie, quorum iudicium non pavesco. Er wünscht sich den Bischof von Chartres. Den verweigert aber der Kanzler: Non tibi datur dominus Carnotensis ... quia longinquus et Romanis negociis occupatissimus. Auch der Bischof von Laon komme nicht in Frage. Am Ende einigt man sich und die Szene schließt in Wohlgefallen.

Nicht die ganze Verhandlung eines anderen Falles, wohl aber ihr wichtigster Teil, ist ebenfalls aus der Zeit Innozenz' II. überliefert. Das Plädoyer wurde vermutlich Anfang Januar 1136 in Pisa vorgetragen. Es ist ein glänzend durchformulierter Text, der den Papst mehrfach unmittelbar anspricht und ihn auch überzeugt hat, denn die umstrittene Kapelle wird der Klägerpartei un-

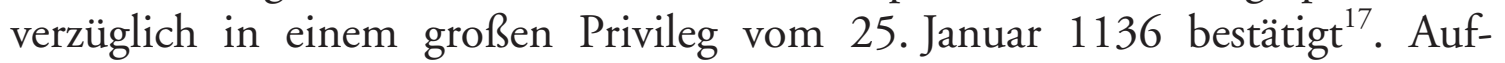
schlußreich schon die unmittelbare Ansprache des Papstes als Weltherrscher: Unice pater orbis et domine. Der Bischof Ulger von Angers referiert die Argumente beider Prozeßparteien, aber von Anfang an macht er sich zum Fürsprecher der armen Regularkanoniker von La Roé gegen die mächtige Abtei Vendôme, die sich in einem höchst bedenklichen Verfahren die besagte Kapelle hat zusprechen lassen, nämlich obwohl La Roé knapp 20 Jahre zuvor von allen zuständigen Instanzen Bestätigungen erhalten hatte. Am Schluß (1117) hatte sogar Papst Paschalis II. seine urkundliche Zustimmung gewährt. Ostentativ hält sie der Bischof dem Papst entgegen: Videte, videte illa, ostendimus et offerimus. Noch bezeichnender wirkt, wie Bischof Ulger die Argumente des

16 Zuvor auch schon in Collectio tripartita 1.13.1 (2. Rez.). Dazu Peter Landau: Gratians unmittelbare Quellen für seine Pseudoisidortexte, in: Fortschritt durch Fälschungen, hg.v. Wilfried Hartmann/Gerhard Schmitz, Hannover 2002 (MGH Studien und Texte 31), S. 184.

17 JL 7755. Das Plädoyer des Bischofs von Angers steht bei Migne PL 179 Sp. 16491654 . 
Gegners zerflückt. Er legt hier dem Abt von La Roé seine Worte in den Mund. Was Vendôme als Urteil zu seinen Gunsten bezeichne, das sei gar kein Urteil, sondern preiudicium oder peregrinum iudicium, et iustum iudicium non fuit quia non fuit factum apud eum quem debuit, nec per eos quos debuit, nec eo modo quo debuit, nec eo loco nec eo tempore, neque circa hos circa quos decuit. Also lauter Verfahrensfehler. Am Schluß heißt es noch einmal in direkter Wendung an den Papst: quia vos estis sustentator pauperum, et pater orphanorum, et advocatus eorum. Vos estis iudex iudicum terre. Sententia vestra definit universas.

Der nächste Fall zeigt zunächst wieder ein mehr anekdotisches Gepräge. Es geht um ein Privileg für die Regularkanoniker von Sainte-Barbe-en-Auge in der Normandie. Wir stehen im Jahr 1147 unter Eugen III. Der Prior von SainteBarbe erscheint an der Kurie mit einem Brief des Grafen von Eu, dem eine lange Besitzliste beigefügt ist. Die in dieser Supplik genannten Güter liegen in der Normandie und in England, sie sollen durch das Privileg des Papstes bestätigt werden. Aus zunächst unklaren Gründen verzögert sich jedoch die Ausstellung. Unser Prior wird immer wieder vertröstet und erfährt die Hintergründe nicht. Bis er am 4. Dezember, dem Fest der Klosterheiligen, der hl. Barbara, persönlich vor dem Papst erscheint und erklärt: Domine, hodie est festum beate Barbare. Memor esto petitionis illius. Antwort Eugens III.: Hodie fiet. Drei Tage später ist das Privileg ausgestellt. Die Chronik von Sainte-Barbe schildert zusätzlich seine Verwendung, denn der wackere Prior reist mit dem kostbaren Dokument unverzüglich nach England und zwingt unter Berufung auf den Papst die widerstrebenden englischen Bischöfe zur Exkommunikation all derer, die noch Güter des normannischen Stiftes in ihrer Hand zurückhalten ${ }^{18}$.

Eine der ausführlichsten Verhandlungsschilderungen des 12. Jahrhunderts gehört 1152 zu dem heftigen, seit langem laufenden Streit zwischen dem päpstlichen Eigenkloster Vézelay und dem Bischof von Autun Heinrich, einem Bruder des Herzogs von Burgund. Der detaillierte Bericht durch den Chronisten der Abtei gewinnt besonderen Wert durch die genau protokollierten Zeugenaussagen, sechs Zeugen, die der Abt aufbietet, zehn Zeugen seitens des Bischofs. In einer Verhandlung muß der Bischof sich so ereifert haben, daß er in Anwesenheit des Papstes erklärte, lieber solle die Abtei Vézelay zerstört werden, als daß sie seinem Befehl nicht gehorchen müsse. Da derselbe Bischof am Ende der Vernehmungen ein ungünstiges Urteil erwartete, erklärte er, weitere für ihn wichtige Zeugen hätten wegen Alter und Schwäche die Reise nach Rom nicht antreten können. Er erwirkte damit Aufschub. Drei delegierte Richter sollten auch diese Zeugen noch vernehmen und ihre Aussagen versiegelt dem Papst

18 Chronique de Sainte-Barbe-en-Auge, ed. René-Norbert Sauvage, Caen 1907, S. 29 36, 40-46. Text des Privilegs Eugens III. in Papsturkunden in Frankreich 2: Normandie, ed. Johannes Ramackers, Göttingen 1937, S. 111-114 Nr. 44, (JL 8964).Vgl. Lohrmann: Kirchengut (wie Anm. 2) S. 90-99. 
zukommen lassen. Die Sache endete seitens des Bischofs und der von ihm angestachelten Bürger von Vézelay mit schweren Übergriffen auf die Abtei, wogegen der Papst in zahlreichen Schreiben Stellung bezog und der Abtei beistand ${ }^{19}$.

Einzuschieben ist hier außerdem der bereits angekündigte Bericht eines langjährigen Dauergastes an der Kurie, Johannes von Salisbury ${ }^{20}$. Seine Erinnerungen gehen auf seine wiederholten Kurienaufenthalte während der Jahre 1148 bis 1152 zurück; eine offizielle Funktion am Papsthof hatte er dabei nicht, weswegen wir ihn durchaus als neutralen Beobachter einstufen können. Die meisten Angelegenheiten erwähnt er nur knapp, so auf dem Konzil von Reims 1148 die Primatsansprüche etlicher Erzbischöfe an Eugen III. und die Versuche einiger Bischöfe, exemte Abteien wieder in ihre Kontrolle zu bekommen. Die Antwort auf diese Eingaben sei in der Regel durch Hinweis auf Verfall der Ansprüche (Präskription) erfolgt, auch hätten die Beklagten darauf verwiesen, daß sie zu dieser Sache nicht geladen seien. Etwas ausführlicher referiert der englische Beobachter den Streit des Erzbischofs von Canterbury mit dem von York; hier zitiert er eine formal begründete Antwort des Papstes wörtlich. Kurz bleiben seine Angaben wieder im Kapitel 4 zur Suspension der Erzbischöfe von Mainz und Köln sowie des Bischofs von Winchester. Gleich zwei ganze Kapitel füllt dagegen die delikate Ehesache des Grafen von Vermandois, bei der es darum ging, Entscheidungen der Vorgängerpäpste aufzuheben; eine unheilvolle Weissagung des Bernhard von Clairvaux zu dieser Sache kam hinzu (Kap. 6 und 7). In dem langen Referat des Johannes über die theologisch begründeten Angriffe gegen den gelehrten Bischof Gilbert von Poitiers spricht Eugen III. an einer Stelle französisch (Gallica utens lingua); die Verhandlung läuft in Paris (Kap. 9-14). In Kapitel 21 (Konzil von Cremona) wehrt der Papst eine Reihe von Primats- und Exemtionsansprüchen ab, diesmal solche von italienischen Prälaten ${ }^{20}$.

Anrührend und aufsehenerregend verläuft schließlich (Kap.41) die von Eugen III. erwirkte Versöhnung des normannischen Grafen Hugo mit dessen Gattin aus dem Hause der Grafen von Tusculum, von der er sich unter Auf-

19 Monumenta Viceliacensia, ed. Robert Buchard Constantijn Huygens, Turnhout 1976 (CChrCM 42), S. 397-408. Dazu die Papstbriefe Nr. 28-50, ebd. S. 314-355. In einem dieser Briefe (Nr. 47) an den Bischof von Autun erinnert sich Eugen III.: de ore tuo audivimus processisse, quia scilicet malebas ut Viceliacense monasterium omnino destrueretur, quam tue non debeat iussioni parere. Der Bericht über die Verhandlung vor Eugen III. wurde schon 1153 an den neuen Papst Anastasius IV. gesandt (ebd. S. 409: qualiter causa ... in presentia Eugenii ventilata per dilatam iurisiudicii diem ... remansisset infecta).

20 S. Anm. 21, Ioannis Saresberiensis Historia pontificalis, ed. Marjorie Chibnall, London 1962. Ausführlicherer Kommentar in der Ausgabe von Iohannis Saresberiensis Historiae pontificalis quae supersunt, ed. Reginald Poole, London/Oxford 1927. 
bietung aller rechtlichen und unrechtlichen Mittel zu trennen versucht hat, falsche Zeugen und die üblichen Bestechungen an der Kurie einbezogen. Der Papst lehnt die Trennung in aller Bestimmtheit ab. Dann erhebt er sich mit Tränen in den Augen, fällt dem Grafen zu Füßen und fleht ihn an, allen Groll zu überwinden und seine Frau wieder zu sich zu nehmen. Selbst seine Mitra ist dem Papst dabei vom Kopf in den Staub gefallen. Dem Grafen, der ihm gerührt zu gehorchen verspricht, schenkt er seinen Ring als signaculum fidei et inite pactionis, quod ego tibi in facie ecclesie tradidi coniugem, et tu eam recepisti in fidem tuam. Diese Szene übertrifft in ihrer Eindringlichkeit die voraufgegangene, am Ende leider mißlungene Versöhnung des Königs von Frankreich mit seiner Gattin Eleonore von Aquitanien durch denselben Papst (Kap. 29). Die Schilderung des normannischen Scheidungsprozesses geht sogar auf verfahrensrechtliche Einzelheiten ein und kommt in dieser Ausführlichkeit in der Italia pontificia, wo sie hingehört, natürlich nicht zur Geltung ${ }^{21}$.

Aus der langen Regierungszeit Papst Alexanders III. ist mir bisher kein auswärtiger Bericht über eine Verhandlung im Konsistorium anläßlich Ausstellung einer strittigen Urkunde bekannt. Dies mag erstaunen: Lief in dieser Zeit alles so geschäftsmäßig, daß es keinen Anreiz zu genauerer Erzählung bot? War der politisch und juristisch höchst versierte Papst gegenüber Bittstellern und Klägern intellektuell so stark überlegen, daß er keine Pannen zuließ? Fehlte in den streng juristischen Untersuchungen das humoristische Element als Anstoß für genauere Berichte oder fehlte nur ein literarisch geübter Beobachter? Erhalten sind immerhin viele hundert Briefe aus den Jahren 1160-1175, die vornehmlich französische Streitsachen betreffen. Auch an Berichten über Verhandlungen mangelt es nicht, aber es sind Angaben des Papstes selbst über seine eigene Vorgehensweise wie etwa in folgendem Fall: „Benevent, (1168-1169) Juni 16. Alexander III. an H(einrich), Erzbischof von Reims. Aus einem Brief des Bischofs (Guido) von Châlons (Deperditum) habe er schon früher erfahren, daß der Überbringer, ein miles $\mathrm{H}$., falsche Zeugen produziert und durch ihr Zeugnis eine Scheidung von seiner Frau erreicht habe. Er und die Zeugen hätten dies dem Bischof eingestanden und von ihm Nachlaß der Missetat gefordert. Daraufhin habe der Papst, wenn er sich recht entsinne, dem Bischof geboten, er solle, falls durch geeignete Zeugen, die keinen Meineid leisten wollten, für ihn feststehe, daß die Zeugen durch Geld zur Aussage veranlaßt worden seien oder zwischen den (ehem. Eheleuten) ein anderer Verwandtschaftsgrad bestehe als sie angäben, dem $\mathrm{H}$. seine Frau zurückgeben und sie zusammenleben lassen, sofern nicht einer von beiden einem anderen sich durch eine Ehe verbunden habe. Dann solle er jenem H. sowie den Zeugen wegen ihres Vergehens eine angemessene Buße auferlegen. Da jedoch der Bischof bisher, wie aus der wiederholten Klage des Mannes, der unter großer Mühe und

21 IP 8 S. 46 Nr. ${ }^{*} 175$. 
Gefahr zum Papst gekommen sei, hervorgehe, nichts davon habe ausführen wollen, habe ihm der Papst durch Schreiben geboten, in derselben Rechtssache gemäß seinem früheren Schreiben unverzüglich und unter Ausschluß einer Appellation vorzugehen. Wolle der Bischof dem vielleicht nicht nachkommen, solle er dies gemäß dem Schreiben, das der Papst dem Bischof habe übermitteln lassen, unverzüglich und ohne die Möglichkeit zur Appellation ausführen“. Das meiste hiervon ist, wie ausdrücklich vermerkt, eigene Erinnerung des Papstes, obwohl dieser an anderer Stelle schreibt (JL 11490), er sei auch nur ein Mensch und erinnere sich nicht an alles ${ }^{22}$.

Nur wenige Hinweise scheinen bisher zur Erklärung des angedeuteten Mangels von Berichten Dritter aus der Zeit Alexanders III. möglich. Zunächst: Der Papst hatte ein phänomenales Gedächtnis. Seine einschränkende Aussage in JL 11490 dient nur dazu, die mühsame Untersuchungsarbeit durch die französischen Bischöfe selbst machen zu lassen. Juristisch ist ihm nicht beizukommen, somit auch nicht viel nach außen zu berichten. Zum anderen: An aufschlußreichen Briefen, die das Getriebe an der Kurie erkennen lassen, ist, wie angedeutet, gerade unter Alexander III. kein Mangel. Sehr viel Licht fällt dort auch auf die Vorgeschichte eines Entschädigungsverfahrens gegen den wegen Veruntreuung eines fremden Depositums angeklagten Abt Ernisius von St. Viktor. Alexander III. überträgt die Untersuchung dieses Falles 1169 dem Erzbischof von Sens und einem künftigen Kardinal, dem Abt Odo von Ours$\mathrm{camp}^{23}$. Seinem Mandat gehen drei Briefe betreffend einen Kanoniker Anselmus von St. Viktor vorauf. Dieser hat versucht, seinem Abt an der Kurie Freunde zu schaffen. Doch der autoritäre Ernisius stößt auch ihn vor den Kopf und empört durch einen ungeschickten Bestechungsversuch die gesamte Kurie ${ }^{24}$.

Im engeren Sinne einschlägig ist eine kurze Mitteilung im letzten dieser Briefe. Dort heißt es, der Kanoniker Anselm habe an der Kurie die Bestätigung

22 Regest von Ludwig Falkenstein für seinen Band Reims innerhalb der Gallia Pontificia nach: Veterum scriptorum et monumentorum historicorum, dogmaticorum, moralium amplissima collectio, ed. Edmond Martène/Ursin Durand, Bd. 2, Paris 1724, Sp. 815 Nr. 229 (JL 11551).

23 Papsturkunden in Frankreich, N. F. 8: Paris I, ed. Dietrich Lohrmann, Göttingen 1989, S. 287 Nr. 108. Als Odo von Ourscamp ein Jahr später die Einladung erhielt, an die Kurie zu kommen, um Kardinal zu werden, antwortete er mit der Wendung: Ut de toto iudicem mundo, pater meus qui me vocavit (Alexander III.) suis collateralibus fecit consedere (Papsturkunden Frankreich, N.F. 7: Nördliche Île-de-France und Vermandois, ed. Dietrich Lohrmann, Göttingen 1976, S. 418 Z. 25). Ähnliche Einschätzungen zur Rolle des päpstlichen Gerichtes 1136 im Plädoyer des Bischofs von Angers (Anm. 17). Die Rolle der Kardinäle im päpstlichen Gerichtsverfahren des 12. Jahrhunderts müßte gesondert behandelt werden. Vgl. u. a. Julius von Pflugk-Harttung, Acta pontificum inedita, Bd. 3, Tübingen 1881, S. 155 Nr. 177 (Urteil von sechs Kardinälen 1139) und weiter unten zu 1195 und 1256.

24 Papsturkunden in Frankreich N.F. 8 (wie Anm. 23) S. 283-286, Nr. 105-107. 
eines Privilegs für das alte Damenkloster Chelles in der Diözese Meaux nur mit größten Schwierigkeiten erreichen können, er übersende dieses nun durch den neuen Archidiakon der Kirche von Meaux ${ }^{25}$. Das ist schon alles, was wir erfahren. Es bleibt der Phantasie des Lesers überlassen, sich vorzustellen, wie die erwähnte maxima difficultas beim Erwerb dieses Privilegs im einzelnen beschaffen gewesen sein mag und mit welchen Mitteln unser wackere Regularkanoniker aus St. Viktor sie überwinden konnte.

Ergiebiger sind die kirchenpolitischen Verhandlungen vor Alexander III. Dafür dürften insbesondere die chronikalischen Nachrichten zum Streit um Thomas Becket etliches Material bieten. Nur eine Szene sei hier herausgegriffen, der Versuch des englischen Königs Heinrichs II., den Papst zur Absetzung des Erzbischofs von Canterbury zu bewegen. Als seine Gesandten dabei auch mit Schmeicheleien und Versprechungen nicht zum Erfolg kamen, gingen sie (AprilMai 1168) zu der Drohung über, der König könne eher zum Glauben des Christenfeindes Nur ad Din, des Vorgängers Saladins, übertreten (Noradini sequeretur errores), als sich länger mit Thomas Bequet als (Erz)bischof von Canterbury abzufinden ${ }^{26}$.

In einem weiteren hochpolitischen Fall geht es Ende 1196 um das Interdikt, das der Erzbischof von Rouen über seine gesamte Kirchenprovinz gelegt hatte, um gegen einen Übergriff des englischen Königs Richard Löwenherz zu protestieren. Das Interdikt erging wegen Übergriffs auf Besitz der Kirche beim Festungsbau des Königs auf einer Seineinsel nahe Les Andelys ${ }^{27}$. Um das Interdikt aufheben zu lassen, appelliert Richard Löwenherz an Papst Cölestin III. Die Chronik des Roger von Howden schildert den Ablauf der Verhandlung im päpstlichen Konsistorium ${ }^{28}$. Zunächst klagt der Erzbischof von Rouen wegen Verletzung des Patrimoniums seiner Kirche durch den Bau der Festung. Die Vertreter des Königs halten dagegen, der König habe sepe et multum Entschädigung angeboten; die Insel sei für ihn unverzichtbar, da an dieser Stelle der König von Frankreich in die Normandie eindringen könne. Beide Parteien unterwerfen sich einer Untersuchung (examini) durch den Papst und die römische Kirche. In ihrer anschließenden Beratung akzeptieren Papst und Kardinäle die Bedürfnisse des Königs bei der Landesverteidigung der Normandie.

25 Ebd. S. 286 Nr. 107.

26 The letters of John Salisbury, Bd. 2: The later letters 1163-1180, ed. W. J. Miller/ C.N.L. Brooke, Oxford 1979 (Oxford Medieval Texts), S. 560 f. Zu dem Interesse König Heinrichs II. für die arabische Wissenschaft vgl. Charles BuRnetT: The Introduction of Arabic Learning into England, London 1997, S. 60 und 101 Anm. 158.

27 Das ist nicht Château-Gaillard. Es erscheint in den Urkunden Richard als Bellum Castrum de Rupe. Vgl. Alexander Cartellieri: Philipp II. August, Bd. 3, Leipzig/Paris 1910, S. 223.

28 Chronica magistri Rogeri de Hovedene, ed. William Stubbs, Bd. 4, London 1871 (RS 51/4), S. $16-18$. 
Sie empfehlen dem Erzbischof, sich mit dem König auszusöhnen, zumal dieser bereit sei, ihn großzügig zu entschädigen. Es kommt zu einer forma pacis ... de consilio domini pape et cardinalium ordinata, die beide Seiten annehmen ${ }^{29}$. Ein umfangreicher Gütertausch wird zunächst vom König beurkundet, nachfolgend vom Erzbischof mit Zustimmung der Bischöfe seiner Kirchenprovinz gebilligt und abschließend am 26. April 1198 durch Innozenz III. mit Insert der Königsurkunde bestätigt ${ }^{30}$. Das Interdikt des Erzbischofs war inzwischen aufgehoben worden.

Abschließend sei für das Ende des 12. Jahrhunderts ein Brief zweier Kardinäle herausgestellt, die 1195 als Auditoren im Auftrag Cölestins III. ein Anliegen der Gräfin von Blois untersuchen sollten. Das Schreiben der Kardinäle zum Streit der Gräfin mit dem Domkapitel von Chartres informiert das Kapitel über den für es günstigen Ausgang. Wie etliche andere Stücke derselben Zeit zeigt es eine ausgeprägt römischrechtliche Terminologie und kündigt mit dem Hinweis auf eine Reihe von ,Artikeln' bereits ein Prozeßverfahren an, das sich voll erst nach 1250 durchsetzen sollte (siehe unten zu 1256). Außerdem zeigt es, daß ein Problem der voraufgehenden Zeit auf dem Weg zu einer institutionell verfestigten Lösung war. Gemeint ist die mangelnde Absicherung Abwesender gegen einseitig erwirkte päpstliche Erlasse, die im 12. Jahrhundert noch häufig vorkommen. In einem bereits laufenden Verfahren vor delegierten Richtern aus Sens, die für Chartres entschieden hatten, versucht die Gräfin sich durch ein einseitig erwirktes Privileg zu schützen. Das Privileg ist bereits bulliert, aber nicht ausgehändigt. Dem Prokurator des Domkapitels von Chartres gelingt es, vor den Kardinälen zu beweisen, daß der Prozeß vor den Delegaten bereits in förmlicher litis contestatio eröffnet worden war. Die Gräfin hatte ferner durch Zeugen auf ,Artikel' des Domkapitels antworten lassen. Dahinter konnte sie prozeßrechtlich nicht mehr zurück. Als deshalb die Kardinäle dem Papst die von ihnen ermittelten Prozeßvorgaben vortrugen, zog Cölestin III. sein Privileg für

29 Erwähnt wird diese Entscheidung Cölestins III. auch bei Radulf von Diceto, Ymagines historiarum, ed. William STubBs, Bd. 2, London 1876 (RS 68/2), S. 158: Haec autem commutatio roborata et confirmata est auctoritate et scripto autentico domini papae ad preces et instantiam domini regis et aliorum magnatum et praelatorum ecclesiae roboranda. Derselbe Chronist hat von Erzbischof Walter von Rouen zum gleichen Streit eine große Zahl weiterer Dokumente erhalten, die er im Wortlaut wiedergibt.

30 Die Register Innocenz' III., 1: 1. Pontifikatsjahr, 1198/1199, bearbeitet von Othmar Hageneder/Anton Haidacher, Graz/Köln 1964 (Publikationen der Abteilung für historische Studien des Österreichischen Kulturinstituts in Rom 2/1), S. 156-160 Nr. 108. Vgl. auch Radulf von Diceto, Ymagines II (wie Anm. 29) S. 160-162. Weitere Chroniken nennt Cartellieri: Philipp II. Augustus, Bd. 3 (wie Anm. 27) S. 139-141. Zur Vorgeschichte vgl. ebd. S. 120, 124-126. 
die Gräfin von Blois sofort zurück und bestätigte statt dessen die Entscheidung der delegierten Richter aus Sens ${ }^{31}$.

Insgesamt zeigt der Überblick über die relativ wenigen Berichte von der Kurie, die wir bisher aus französischer Überlieferung im 12. Jahrhundert haben, noch deutliche Schwächen in der Organisation der Vergabe päpstlicher Gerichtsurkunden und Privilegien. Die Prüfung der gegensätzlichen Interessen und Rechtsansprüche gelingt teilweise nur unvollkommen, da die streitenden Parteien nicht gleichzeitig zur Stelle sind oder die Impetranten es bewußt darauf anlegen, falsche oder lückenhafte Angaben zu machen ${ }^{32}$. So muß unbedingt eine Institution geschaffen werden, die vor der Übergabe der Urkunden an einen Impetranten der Gegenseite Gelegenheit gibt, Einspruch zu erheben und die Aushändigung der Urkunde aufzuhalten. Dies geschieht relativ schnell unter dem jungen rechtserfahrenen Papst Innozenz III.

\section{Berichte des 13. Jahrhunderts}

Kein Text erlaubt es, diese neue Praxis zusammen mit etlichen Einzelberichten über Verhandlungen vor Innozenz III. im Konsistorium besser kennenzulernen, als der soeben neu edierte Text des rechtskundigen Mönches Thomas von Evesham in seiner «Historia abbatie Eveshamensis» ${ }^{33}$. Quantitativ wie qualitativ wird hier ein ganz neues Stadium der Berichterstattung von der Kurie erreicht. Wir hören von erfolgreichen, aber auch verzögerten und gescheiterten Petitionen, von zahlreichen Aktionen vor Papst und Kardinälen, erfahren, auf welche Weise der Papst erkennen läßt, wie er das Verfahren zu lenken gedenkt, wie er eine Zweiteilung des Verfahrens vornimmt, wie er die Einlassungen der beiden Prokuratoren knapp kommentiert, nötigenfalls auch abrupt unterbricht, wenn sie von der erörterten Rechtsfrage zu anderen Erwägungen übergehen. Nicht zuletzt zeigt sich die geradezu prozeßentscheidende Bedeutung der Kreditauf-

31 Papsturkunden in Frankreich, N.F. 6, ed. Johannes Ramackers, Göttingen 1958, S. 267-272 Nr. 206 und 208.

32 Bei Roger von Howden, Chron. 4 (wie Anm. 28) S. 15, sind als Briefe dieser Art auch die „vielen falschen Briefe“ einzustufen, die 1196 ein Kleriker des Erzbischofs von York an der Kurie erwirkt hatte. Als er darauf, noch in Rom, auf den Tod erkrankte, gestand er sein Vergehen ein. Weiteres zum Problem der erschlichenen Briefe im späteren 12. Jahrhundert hat Herde: Beiträge (wie Anm. 4) S. $92 \mathrm{f}$.

33 Thomas of Marlborough, History of the Abbey of Evesham, ed. and transl. Jane E. Sayers/Leslie Watkiss, Oxford 2003 (Oxford Medieval Texts), Buch II S. 266-314 $\$ \$ 263-316$. Vgl. Jane SAYERs: „Original“, Cartulary and Chronicle: the Case of the Abbey of Evesham, in: Fälschungen im Mittelalter. Internationaler Kongreß der Monumenta Germaniae Historica München, 16.-19. September 1986, Hannover 1998 (MGH Schr. 33/4), S. 371-395. 
nahme durch die Parteien bei den sogenannten mercatores Romani $i^{34}$. Nur wer dank solcher Kredite die teuersten Advokaten bezahlen kann, hat eine Chance, sich in der Verhandlung durchzusetzen. Im einzelnen kann ich das hier nicht verfolgen, empfehle deshalb jedem, der noch nicht Gelegenheit dazu hatte, die Lektüre dieses englischen Berichtes wärmstens.

Ein französischer Prozeßbericht vom Juli 1256, den ich vor etwa 35 Jahren in Paris entdeckt habe und jüngst zusammen mit Olivier Guyotjeanmin in der Revue du Nord veröffentlichen konnte, soll hier den Abschluß bilden ${ }^{35}$. Er enthält nicht nur als Hintergrundinformation neue ergänzende Nachrichten zu einem Volksaufstand der Römer im April-Mai dieses Jahres unter Alexander IV. Drohungen gegen einige adlige römische Kardinäle führten damals zur Befreiung des Senators Brancaleone. Wichtig sind hier vor allem die Einzelstadien des Prozesses. Dieser kann erst beginnen, nachdem die Kurie Anfang Juni nach Anagni geflüchtet ist. Was dann folgt erinnert zum Teil an den oben referierten Fall Oudenburg gegen Saint-Médard. Der nun freilich viel längere Bericht des Abtes von Saint-Éloi in Noyon, der sich gegen die Übermacht des Domkapitels von Noyon zur Wehr setzt, schildert zunächst die bessere Ausgangsposition der Gegenpartei: Mehrere päpstliche Kapläne sind zugleich Kanoniker des Domkapitels Noyon. Sie unterstützen selbstverständlich dessen Position und haben jederzeit Zugang zum Papst. Dazu sind mehrere ihrer vielen Advokaten in Anagni Neffen des hochangesehenen (erst vor einigen Jahren verstorbenen) Kardinalbischofs Pietro da Collemedio, während der arme Abt von Saint-Éloi sich gerade einen Advokaten leisten kann, der dazu noch teuer ist, denn er ist von Rom nach Anagni mitgekommen. Der Abt muß dort für ihn ein ganzes

34 Thomas von Marlborough (wie Anm. 33) S. $344 \$$ 358. Schon Abt Hariulf vor Innocenz II. (vgl. oben zu 1141) notierte: sed vereor, ne sacculus inanis victum neget diu moranti. „Aber ich fürchte, mein leerer Geldbeutel wird mir einen längeren Aufenthalt an der Kurie nicht ermöglichen“. Gute Bemerkungen hierzu wie zu dem gesamten Prozeß bei Harald Müller: Der Exemtionsprozeß des Klosters Evesham an der päpstlichen Kurie in den Jahren 1202-1206, Magisterarbeit RWTH Aachen WS 1990-1991, S. 122131 (finanzielle Aspekte). In die Zeit Innocenz' III. gehört auch die Klage des «Dialogus inter euntem ad curiam et venientem a Roma de malis moribus curie», wo es satirisch u. a. heißt: Plena rancoribus est tota curia./ Prodest datoribus sola pecunia./ In cardinalibus viget consilium./ Si donas talibus, vocant te filium, ed. Herde: Beiträge (wie Anm. 4) S. $247-251$.

35 Olivier Guyotjeannin/Dietrich Lohrmann: Labbé de Saint-Éloi de Noyon en cour de Rome (1256), in: Revue du Nord 86 (2004) S. 681-696. Gesamtübersicht über den Prozeß nach Paris BnF ms. lat. 13777 bei Olivier Guyotjeannin: Les reliques de saint Éloi à Noyon: procès et enquêtes du milieu du XIII ${ }^{\mathrm{e}}$ siècle, in: Revue Mabillon n. s. 1 (1990) S. 57-110. 
Haus samt Stallungen für die Pferde mieten. Zum Glück hat ihm sein Konvent eine Vollmacht zur Kreditaufnahme mitgegeben ${ }^{36}$.

Dies war für den Abt eine eher ungünstige Ausgangslage. Beim ersten Konsistorium ergibt sich jedoch eine Vertauschung der Rollen von Kläger und Beklagtem, weil die Vertreter des Domkapitels bei Beginn der Verhandlung gleich ungestüm vorpreschen ${ }^{37}$. Der Abt von Saint-Éloi weiß diesen Fehler geschickt zu nutzen. Während seine Gegner, so führt er aus, nur die Fortsetzung des laufenden Untersuchungsverfahrens in Noyon durch früher ernannte Richter aus Soissons erreichen wollten, forderte er für sein Kloster eine Verhandlung zur Hauptsache, de natura principalis negotii: Das eigentliche Skandalon des Streites um die Reliquien müsse ausgeräumt werden, also seien keine weiteren Ausflüchte, Zänkereien und Gehässigkeiten (diffugia, rixae und odia) zuzulassen. Die seien abzuwenden (avertenda). Auch dürften keine weiteren unnützen Kosten für die streitenden Kirchen entstehen, im päpstlichen Gericht gehe es doch darum, den Hauptstreitpunkt, das principale negotium, abzuschließen. Mit dieser Argumentation gewinnt der Abt bei Papst und Kardinälen Sympathien. Die Sitzung wird unterbrochen und schon am übernächsten Tag weitergeführt. Zwischendurch bleibt Zeit für Lobbyarbeit unter den Kardinälen. Daran läßt es unserer wackerer Abt nicht fehlen: „Wir eilten durch die Reihen der Kardinäle und informierten sie in unserem Sinne" (per cardinales discurrimus ... juxta motum nostrum informantes eosdem).

Im zweiten Konsistorium nach Beratung mit den Kardinälen legt Alexander IV. eingangs sofort den künftigen Kurs fest. 1. Das laufende Untersuchungsverfahren vor Richtern aus Soissons wird eingestellt; die bisherigen Prozeßschritte und Zeugenaussagen zugunsten des Domkapitels von Noyon sind damit nichtig. 2. Ein neues Delegationsmandat soll an eine unvoreingenommene Richterpersönlichkeit im Königreich Frankreich gehen, an einen Unverdächtigen, der omni suspicione careat. 3. Der neue Untersuchungsauftrag zielt auf die Klärung des Verbleibs der Reliquien des hl. Eligius, ubi sit, aber auch auf das, was es mit der eigentlichen Grabstätte auf sich hat, mit der Translation in die Kathedrale, den Wundern und dem Pilgerkult. 4. Außer Zeugenaussagen sind ausdrücklich zu nutzen auch hystoriae, cronicae, legendae, libri antiqui et aliae probationes legitimae.

36 Text der Vollmacht im Anhang. Sehr aufschlußreiche Dokumente zur Kreditaufnahme durch Prokuratoren an der Kurie 1257-1259 veröffentlicht Werner MaLECZEK: Erzbischof Ulrich von Salzburg (1257-1265), die päpstliche Kurie und Bankiers aus Rom, Florenz und Venedig, in: Tirol - Österreich - Italien. Festschrift für Josef Riedmann zum 65. Geburtstag, hg.v. Klaus Brandstätter/Julia Hörmann, Innsbruck 2005 (Schlern-Schriften 330), S. 405-439.

37 Etwas ähnliches zeigt Thomas von Evesham auch im Exemtionsprozess gegen den Bischof von Worcester, ed. SAyers-Watkiss (wie Anm. 33) S. $278 \$ 280$. 
Die Liste der zugelassenen Beweismittel wird hier vom Papst im Interesse des Klosters stark erweitert. Auf diese erweiterten Beweismittel hat 1985 bereits Erika Laquer hingewiesen ${ }^{38}$. Wichtiger für unser Thema scheint eine Innovation bei den Auflagen für das Vorgehen des neu delegierten Richters. Er soll vorgehen secundum articulos. Gemeint sind Prozeßartikel, die von beiden Parteien auszuformulieren und sub bulla dem Mandat an den Richter beizufügen sind. Erwähnt fanden wir solche Artikel bereits im oben behandelten Rechtsstreit der Gräfin von Blois gegen das Domkapitel von Chartres. In der Prozeßdokumentation aus Saint-Éloi haben sich diese Artikel (31 für jede Partei) im Wortlaut erhalten. Hinzu treten Stellungnahmen der jeweiligen Gegenpartei zu den einzelnen Artikeln. Diese sind im Konsens unter Leitung eines Kardinals zu akzeptieren, andernfalls werden sie gestrichen. Vor allem müssen diese Prozeßartikel zur Sache gehören, sie müssen pertinentes sein; articuli impertinentes sind nach Vorgabe der zeitgenössischen ,Regulae iuris' auszuscheiden. Die Aufsicht über diesen Vorgang obliegt an der Kurie einem erfahrenen Juristen, der in vielen Prozessen eine Rolle gespielt hat, dem englischen Zisterzienserkardinal Johannes von Toledo ${ }^{39}$.

Das gesamte Verfahren, das hier in einer frühen Phase aus der Praxis dokumentiert ist, entspricht, wie ich schon andeutete, einer juristischen Innovation des 13. Jahrhunderts, die am Ende des 12. Jahrhunderts schon vorbereitet $\operatorname{war}^{40}$. Mit den Artikeln grenzte jede Partei im vorhinein den Rahmen dessen ein, was sie in der richterlichen Untersuchung zu beweisen vorgab. Es waren Beweisanträge, die dem delegierten Richter seine Aufgabe der Zeugenvernehmung erleichtern sollten. Die Rechtsgeschichte kennt das Verfahren unter der Bezeichnung Positionalverfahren (positiones probandae im Sinne von articuli pro-

38 Erika Laquer: Ritual. Literacy and Documentary Evidence: Archbishop Eudes Rigaud and the Relics of saint Éloi, in: Francia 13 (1985) S. 625-637.

39 Näheres bei Dietrich Lohrmann: Delegatio cum annexis articulis et questionariis. Die prozessrechtliche Wende im Streit um die Reliquien des hl. Eligius von Noyon, in: Grosse: Acte pontifical (wie Anm. 14) S. 229-264. Nur drei Tage nach Ausstellung des neuen Delegationsmandates für den Erzbischof von Rouen folgt eine bemerkenswerte Schilderung von Verfahrensschritten nach römischem Prozeßrecht in der Entscheidung eines Streites um Nutzungsrechte zwischen der Zisterzienserabtei Igny (Diöz. Reims) und der Dorfgemeinde Montigny durch den Kardinal Stephan von Präneste vom 29. Juni 1256: Les Registres d'Alexandre IV, Bd. 3, ed. Auguste Coulon, Paris 1953, S. 47 48.

40 Siehe Anm. 31. Klagelibelli, Petitionen und Zeugenaussagen der Parteien sind spätestens seit dem 12. Jahrhunderts auch aus Prozessen in Italien bekannt. Vgl. u. a. Margarete Pollock: Der Prozess von 1194 zwischen Orvieto und Savona um das Val di Lago, in: QFIAB 70 (1990) S. 46-150; im Anhang dort u.a. die Aussagen von 23 Zeugen für Savona und 34 Zeugen für Orvieto. Dazu Johannes Fried: Der Schleier der Erinnerung. Grundzüge einer historischen Memorik, München 2004, S. 178-183, der sich für die Zuverlässigkeit der „steinalten“ Zeugen interessiert. 
bandi) ${ }^{41}$. So sind all diese Artikel der Parteien am Anfang auch ausformuliert: volumus probare.

Schließlich noch ein Wort zur Auswahl des delegierten Richters in der fortgeschrittenen Phase des über 30-jährigen Prozesses von Noyon, in der alles noch einmal neu auf dem Spiel steht. Es ist wieder eine von den besonders eindrücklichen, filmreifen Kurzszenen: Am Ende des zweiten Konsistoriums nimmt sich der Papst den wackeren Abt von Saint-Éloi zur Seite und flüstert ihm zu, ob für ihn der Erzbischof von Rouen als Richter akzeptabel sei. Der Abt, zu Dank verpflichtet, zögert bei dieser wichtigen Frage, lehnt aber nicht ab wie seinerzeit 1141 der Abt von Oudenburg es wegen Befangenheit der Richter getan hatte. Nein, er überlegt und antwortet vorsichtig, er habe keinen Befangenheitsgrund einzuwenden, keine causa suspicionis. So wird der vielbeschäftigte Erzbischof Odo von Rouen tatsächlich ernannt und hat das zweifelhafte Vergnügen, sich volle fünf Jahre lang mit dem verzwickten Streit um die Reliquien des heiligen Eligius auseinandersetzen zu dürfen ${ }^{42}$.

\section{Anhang}

Vollmacht des Konvents von Saint-Éloi in Noyon für Abt Johannes zur Kreditaufnahme während seiner Romreise.

Noyon, 1256 (Februar März)

Paris BnF, ms. lat. 13777 fol. 297rv (Guyotjeannin, Reliques, wie Anm. 35, S. 101 n. 199).

$\mathrm{Zu}$ den Texten des zugehörigen Prozeßberichtes und des neuen päpstlichen Delegationsmandates samt Anlagen für den delegierten Richter, Erzbischof Odo von Rouen, siehe oben Anm. 36, Weiteres zu Kreditaufnahmen an der Kurie in Anm. 34 und 36.

Universis Christi fidelibus ad quos presentes littere pervenerint, Petrus prior Sancti Eligii Noviomensis et conventus eiusdem loci salutem in Domino. Universitati vestre tenore presentium innotescat, quod cum vir religiosus Jo-

41 Thomas Wetzstein: Heilige vor Gericht. Das Kanonisationsverfahren im europäischen Spätmittelalter, Köln-Weimar/Wien 2004 (Forschungen zur kirchlichen Rechtsgeschichte und zum Kirchenrecht 28), S. 33-56. Für wertvolle Hinweise in diesem Zusammenhang danke ich Martin Bertram, Rom, und Harald Müller, Mainz.

42 Diese vorläufig letzte Phase des Prozesses ist noch nicht näher untersucht. Gesamtübersicht siehe bei Guyotjeannin: Reliques (wie Anm. 35). Vgl. auch Laquer (wie Anm. 38). 
hannes permissione divina noster abbas providus et fidelis, lator presentium, sui et nostri monasterii necessitatibus et angariis pio compatiens affectu, multimoda precum nostrarum instantia per nos fuit ductus, ut sollicitudinibus, periculis et labori pro nobis totaliter se exponens iter arripiat ad Romanam curiam transeundi, nos fidei et diligentie ipsius commendabili pro tot laboribus et angustiis pie non immerito condolentes, et honori eius quantum possumus deferre volentes, et caventes ne aliqua sibi desint ad sumptus utiles et honestos et ad omnimodas expensas pro nostris negotiis promovendis secundum eius arbitrium faciendas, attendentes etiam nobis et sibi fovere commodius per ipsum pecuniam mutuo recipere et in regno Francie reddere, quam cum tot laboribus et sumptibus et per tot pericula transportare, ipsum patrem et dominum nostrum constituimus syndicum seu actorem et ei liberam concedimus potestatem ad recipiendum pro nobis et nomine nostro pecuniam mutuo usque ad quantitatem quam ad nostras utilitates provehendas et nostra negotia procuranda viderit competentem, et ad pecuniam ipsam erogandam prout viderit expedire, et ad obligandum bona monasterii nostri pro dictis mutuis ab ipso contrahendis, promittentes sub ipoteca rerum nostrarum nos reddituros mutua que contraherit cum eorum accessibus in locis terminis et conditionibus queque voluerit inter ipsum et mutuatores quoslibet a quibus duxerit mutua contrahenda. Ratum et gratum habituri quicquid idem patet et abbas noster super predictis omnibus duxerit faciendum. In cuius rei testimonium et munimen presentes litteras eidem tradidimus sigilli nostri conventus impressione munitas. Dat. et cetera.

\section{Riassunto}

Sui processi, svoltisi nei secoli XII e XIII presso la curia, si hanno di norma notizie solo attraverso il risultato, la conferma definitiva del papa e/o le decisioni $o$ indagini preliminari da parte di giudici delegati. Niente invece viene fuori sul dibattimento vero e proprio nel concistorio, a parte la breve annotazione che la questione è stata esaminata davanti al papa e ai cardinali. Nell'Italia pontificia si trovano pertanto solo pochi accenni finora raccolte di questo tipo. Le cronache e gli archivi francesi offrono alcune scene dettagliate e interessanti che qui vengono analizzate, e dove appaiono anche alcuni tratti umani e spiritosi dei papi medievali. Si inizia con il famoso processo contro l'arcivescovo Manasse di Reims e si termina nel 1256 con una delegazione, ottenuta con fatica, dell'arcivescovo Odo di Rouen per il maxiprocesso intorno alle reliquie di S. Eligio di Noyon. In mezzo si hanno importanti innovazioni relative al diritto processuale, la creazione dell'Audientia litterarum contradictarum e l'introduzione del procedimento posizionale per l'interrogatorio dei testimoni. Vengono presentati pure alcuni processi di forte valenza politica. Sarebbero da esaminare più da 
vicino le questioni dei costi e le circostanze, in cui avvenivano le accensioni di credito presso i mercatores Romani. Una relativa procura viene riprodotta in appendice. 